\title{
Production of cellulosic organic acids via synthetic fungal consortia
}

\author{
Scott A. Scholz ${ }^{1}$ (D) | Ian Graves ${ }^{2}$ | Jeremy J. Minty ${ }^{2}$ | Xiaoxia N. Lin ${ }^{2,3}$
}

${ }^{1}$ Graduate Program in Cellular and Molecular Biology, University of Michigan, Ann Arbor, Michigan

2 Department of Chemical Engineering, University of Michigan, Ann Arbor, Michigan

${ }^{3}$ Department of Biomedical Engineering, University of Michigan, Ann Arbor, Michigan

\section{Correspondence}

Xiaoxia N. Lin, Department of Chemical Engineering, University of Michigan, Ann Arbor, MI 48109.

Email: ninalin@umich.edu

Funding information

National Institutes of Health, Grant number: T-32-GM007315

\begin{abstract}
Consolidated bioprocessing (CBP) is a potential breakthrough technology for reducing costs of biochemical production from lignocellulosic biomass. Production of cellulase enzymes, saccharification of lignocellulose, and conversion of the resulting sugars into a chemical of interest occur simultaneously within a single bioreactor. In this study, synthetic fungal consortia composed of the cellulolytic fungus Trichoderma reesei and the production specialist Rhizopus delemar demonstrated conversion of microcrystalline cellulose (MCC) and alkaline pre-treated corn stover (CS) to fumaric acid in a fully consolidated manner without addition of cellulase enzymes or expensive supplements such as yeast extract. A titer of $6.87 \mathrm{~g} / \mathrm{L}$ of fumaric acid, representing $0.17 \mathrm{w} / \mathrm{w}$ yield, were produced from $40 \mathrm{~g} / \mathrm{L} \mathrm{MCC}$ with a productivity of $31.8 \mathrm{mg} / \mathrm{L} / \mathrm{hr}$. In addition, lactic acid was produced from MCC using a fungal consortium with Rhizopus oryzae as the production specialist. These results are proof-of-concept demonstration of engineering synthetic microbial consortia for CBP production of naturally occurring biomolecules.
\end{abstract}

\section{KEYWORDS}

consolidated bioprocessing, fumaric acid, lignocellulosic biomass, synthetic consortia

\section{1 | INTRODUCTION}

Lignocellulosic biomass is an attractive substrate for bioconversion into industrial chemicals because it is the most abundant terrestrial renewable bio-feedstock on earth. As a non-edible plant substrate, lignocellulose can be produced as agricultural and forest residues, which do not require massive land use changes. There are also strong social motivations for using lignocellulosic biomass as a replacement for edible substrates currently used for industrial bioconversions, such as corn and simple sugars (Dunn, Mueller, Kwon, \& Wang, 2013). However, due to the recalcitrant nature of lignocellulose to enzymatic hydrolysis, it has not been widely used as an industrial feedstock (Carroll \& Somerville, 2009). Consolidated bioprocessing (CBP) has been widely discussed as a strategy for improving the efficiency of converting lignocellulosic biomass into industrial biochemicals (Brethauer \& Studer, 2014; Kawaguchi, Hasunuma, Ogino, \& Kondo,
2016; Parisutham, Kim, \& Lee, 2014). In CBP enzyme production, enzymatic hydrolysis of lignocellulose and conversion of resulting sugars to biochemicals occur simultaneously in a single reaction vessel, resulting in significant potential cost savings (Olson, McBride, Shaw, \& Lynd, 2012). One approach for CBP has been to genetically engineer a single microorganism to produce cellulases and convert sugars into desired biochemicals. However, the efficiency of cellulase production, secretion and activity remains a major obstacle to this approach (den Haan, van Rensburg, Rose, Görgens, \& van Zyl, 2015; Lambertz et al., 2014). Additionally, the requirement for tremendous new efforts of engineering a single microorganism to produce a new chemical of interest has made this approach difficult from a practical standpoint. Recently, a number of CBP systems have been designed to combine more than one microorganism. In these approaches, two or more microorganisms are cultured together, typically dividing the tasks of hydrolysis and biochemical production between microbial specialists. 


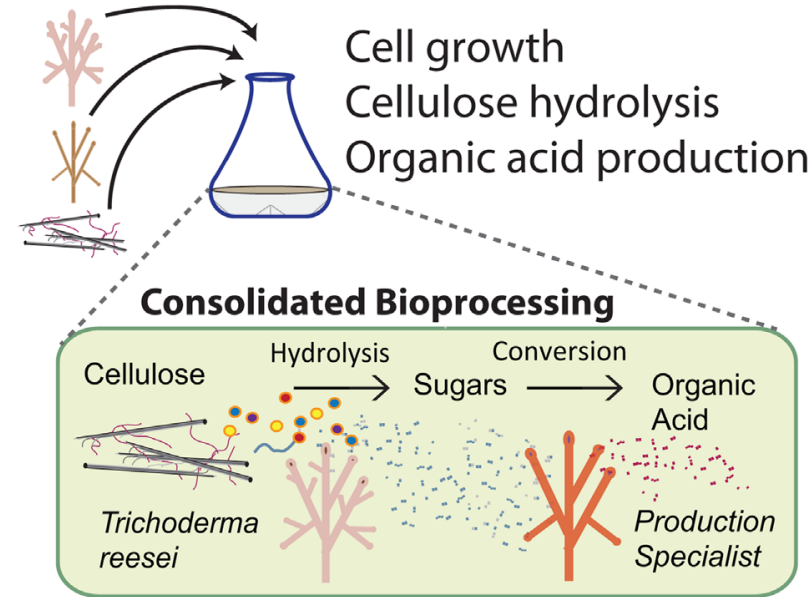

FIGURE 1 Overview of CBP system for organic acid production. $T$. reesei and the production specialist organism are inoculated simultaneously into the production medium. Starting from a low initial cell density, all processes including cell growth, cellulase production, cellulose hydrolysis and conversion of sugars into organic acids occur in a single reaction vessel

These systems are more modular, allowing different chemicals to be produced without major genetic redesigns. Several groups have successfully designed synthetic consortia-based CBP strategies for producing ethanol (Brethauer \& Studer, 2014; Goyal, Tsai, Madan, DaSilva, \& Chen, 2011; Kim, Baek, Lee, \& Hahn, 2013). A synthetic consortium CBP system has also been designed for the production of isobutanol from lignocellulosic biomass by pairing the cellulolytic fungus Trichoderma reesei with an engineered isobutanol-producing Escherichia coli strain (Minty et al., 2013).

For the present work, we designed synthetic fungal consortia to produce fumaric and lactic acids from cellulose and lignocellulosic biomass. Our preferred cellulolytic specialist was T. reesei because of its extensively documented efficient cellulase enzyme production and conversion of cellulose into monomeric sugars in minimal media (Figure 1) (Peterson \& Nevalainen, 2012). Therefore, production specialist candidates were assessed based on efficient bioconversion of sugars into organic acids in similar minimal media. Factors such as temperature, aeration, and culture conditions were considered for compatibility. Finally, production specialists previously demonstrating the highest yields and titers of organic acids were prioritized. Using these criteria, we selected Rhizopus delemar (fumaric acid) and Rhizopus oryzae (lactic acid) as production specialists for synthetic consortia CBP. In each CBP system, the hydrolysis, and production processes occur simultaneously. Carbon is liberated from cellulose by cellulase enzymes produced by $T$. reesei and the resulting sugars are immediately converted into organic acids by the production specialist in the same bio-reactor (Figure 1). Our successful design and implementation of synthetic consortia CBP for production of fumaric and lactic acid represents a significant step towards establishing a robust, versatile, and modular platform technology for consortia-based CBP conversion of lignocellulosic biomass to a wide variety of biochemicals.

A defined minimal medium Rhizopus-Trichoderma co-culture medium (RTco) was formulated to allow both cellulose hydrolysis and fumaric acid production without the need for supplementation with expensive components such as yeast extract. $R$. delemar switches from growth to fumaric acid production phase when nitrogen is no longer available in culture media (Ding, Li, Dou, Yu, \& Huang, 2011). Therefore, RTco was formulated with a nitrogen concentration that is $12.5 \%$ of those commonly used for $T$. reesei growth and cellulase production (Juhász, Szengyel, Réczey, Siika-Aho, \& Viikari, 2005; Minty et al., 2013). Under these conditions, both fungi are expected to grow until nitrogen becomes limiting in the production medium, at which point growth and cellulase production would cease, while fumaric acid production begins. Each fungal strain was first characterized in monocultures with the RTco medium. Monocultures of $T$. reesei grown on $40 \mathrm{~g} / \mathrm{L}$ microcrystalline cellulose (MCC) in RTco medium efficiently accumulated glucose as expected (Figure 2a). Under the proposed consortia CBP conditions $22 \mathrm{~g} / \mathrm{L}$ of glucose is produced from MCC at a productivity of $65 \mathrm{mg} / \mathrm{L} / \mathrm{hr}$ after $336 \mathrm{hr}$ fermentation time. Monocultures of $T$. reesei were also grown on $20 \mathrm{~g} / \mathrm{L}$ alkaline pre-treated corn stover (CS) in RTco medium. The CS utilized is composed of $47.8 \%$ and $21.2 \%$ of non-soluble glucan and xylan by weight, respectively.
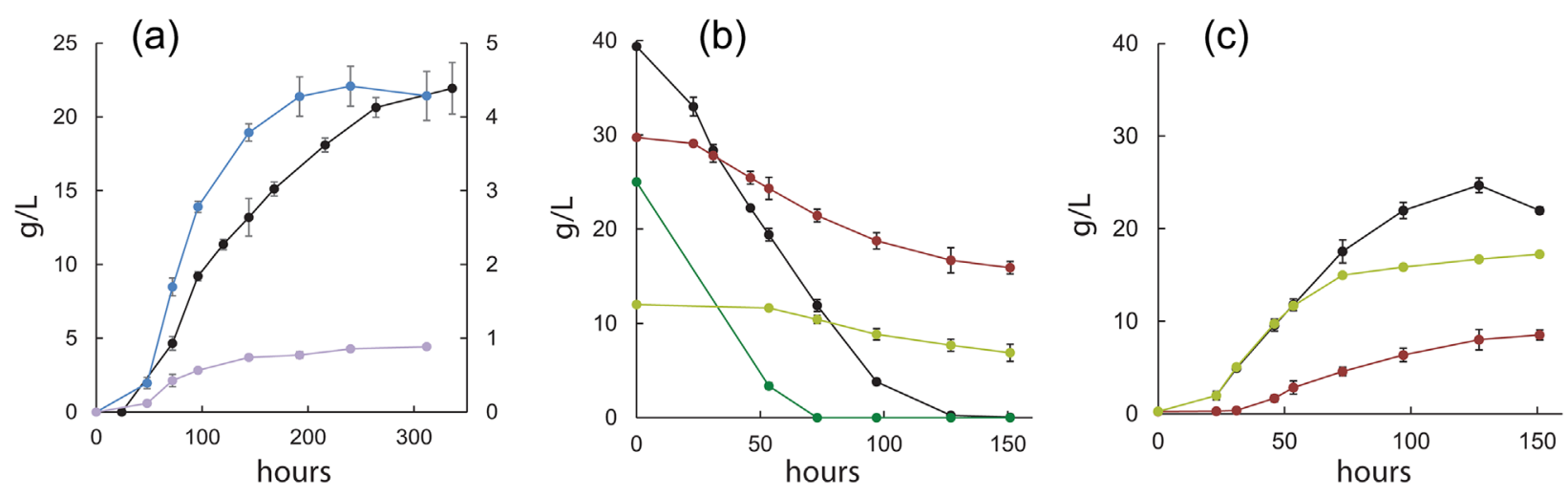

FIGURE 2 Monocultures exhibit efficient specialist activities in RTco medium formulated for co-culture. (a) Sugar accumulation by T. reesei in two monoculture experiments: glucose (Black, left y-axis) from $40 \mathrm{~g} / \mathrm{L} \mathrm{MCC}$; glucose (Blue, right y-axis) and xylose (Purple, right y-axis) from $20 \mathrm{~g} / \mathrm{L}$ alkaline pretreated corn stover. (b) R. delemar monoculture can utilize pure glucose (Black), pure xylose (Red), or a mix of glucose (Dark green) and xylose (Light green) in RTco medium. (c) R. delemar production of fumaric acid from sugar substrates corresponding to (b). Data points in light green represent fumaric acid production from a mix of glucose and xylose 
Glucan and xylan account for $95 \%$ of the carbohydrates in the CS. It was observed that $4.4 \mathrm{~g} / \mathrm{L}$ glucose accumulated from hydrolysis of the CS, representing $41 \%$ of the theoretical maximum yield from glucan, while $0.86 \mathrm{~g} / \mathrm{L}$ xylose accumulated, representing $15 \%$ of the theoretical maximum yield from xylan. Total sugar productivity was $22 \mathrm{mg} / \mathrm{L} / \mathrm{hr}$ over the course of $240 \mathrm{hr}$. R. delemar monoculture efficiently consumed $40 \mathrm{~g} / \mathrm{L}$ glucose in RTco medium (Figure $2 \mathrm{~b}$ ) to produce $22 \mathrm{~g} / \mathrm{L}$ fumaric acid (Figure $2 \mathrm{c}$ ), representing a yield of $0.55 \mathrm{w} /$ w and a productivity of $153 \mathrm{mg} / \mathrm{L} / \mathrm{hr}$. The theoretical maximum yield of fumaric acid is two moles per mole of glucose upon fixation of two moles of $\mathrm{CO}_{2}$ in a reductive carboxylation pathway. By weight, $1.29 \mathrm{~g}$ of fumaric acid would be produced per gram of glucose. However, this production pathway would not allow for production of ATP and requires $\mathrm{CO}_{2}$ fixation (Roa Engel, Straathof, Zijlmans, van Gulik, \& van der Wiele, 2008). Nitrogen concentration controls the tradeoff between cell growth and fumaric acid production (Ding et al., 2011). With minimal glucose substrate directed to cell growth, yields of up to $0.85 \mathrm{w} / \mathrm{w}$ from glucose have been reported. Consistent with previous observations with similar fungal strains (Kautola \& Linko, 1989), $R$. delemar was also capable of utilizing xylose as the sole or a portion of the carbon source in RTco medium to produce fumaric acid, albeit more slowly than on glucose. Additionally, $R$. delemar grown on medium containing mixed glucose and xylose demonstrated usage of both sugars and accumulation of fumaric acid (Figures $2 b$ and $2 c$ ). Results described above demonstrate the compatibility of T. reesei and $R$. delemar to be grown together for consolidated conversion of cellulose to fumaric acid in RTco medium.

The tradeoff between fumaric acid production rate and yield from glucose by $R$. delemar can be controlled by nitrogen concentration (Ding et al., 2011). R. delemar monocultures with high nitrogen concentrations lead to more $R$. delemar cell growth and higher subsequent production rates of fumaric acid, but achieve lower final yields. Likewise, in consortium CBP the nitrogen concentration can also control the amount of carbon that is utilized for cell growth versus carbon directed towards producing fumaric acid. Therefore, nitrogen concentration should be a key parameter for optimizing the T. reesei- $R$. delemar consortium CBP system. To test whether the proposed fungal consortium could indeed produce fumaric acid from cellulose and whether nitrogen can control production dynamics as expected, we monitored consortium performance in RTco medium with three nitrogen concentrations. Nitrogen concentration variation led to different culture dynamics and production titer, yield and productivity (Figure 3). Production medium with a low $5.88 \mathrm{mM}$ nitrogen concentration allowed for relatively high amounts of glucose accumulation (Figure 3a) and slow fumaric acid production, eventually achieving 0.148 yield by MCC weight and $16.6 \mathrm{mg} / \mathrm{L} / \mathrm{hr}$ productivity (Figure $3 b$ ). Comparatively, an intermediate nitrogen concentration of $11.76 \mathrm{mM}$ led to slow initial glucose accumulation and a decrease in glucose concentration at later time points, due to conversion into fumaric acid. Fumaric acid production under intermediate nitrogen concentration condition outperformed the other nitrogen concentrations tested in terms of yield $(0.17$ by weight), productivity $(31.8 \mathrm{mg} / \mathrm{L} / \mathrm{hr})$ and titer $(6.87 \mathrm{~g} / \mathrm{L})$. In medium with the highest nitrogen concentration tested, $23.5 \mathrm{mM}$, almost no glucose accumulation was detected, fumaric acid accumulation was delayed, and the fumaric acid yield reached only 0.137 by weight. These results are consistent with a greater proportion of carbon being allocated for fungal growth under higher nitrogen conditions. We note that under optimal process control only low concentrations of glucose would accumulate, indicating that the rate of sugar liberation from MCC by $T$. reesei closely matches the rate of sugar conversion into fumaric acid by $R$. delemar without actually limiting conversion due to sugar limitation. Promising future work for further engineering this consortium include developing new strategies to differentially regulate the growth of the two consortium members. We also designed a lactic acid-producing consortium CBP system by replacing $R$. delemar with $R$. oryzae (NRRL 395) and carried out initial experiments using the same nitrogen concentration in TMM medium. Lactic acid titer of $4.4 \mathrm{~g} / \mathrm{L}$, representing a $0.11 \mathrm{w} / \mathrm{w}$ yield and $16.7 \mathrm{mg} / \mathrm{L} / \mathrm{hr}$ productivity, was achieved (Figure 3c). Due to observations that lactic acid may be degraded by $T$. reesei (Data not shown), we did not pursue further characterization of this consortium in the present study.

Next, we investigated consortium performance on alkaline pretreated CS. Lignocellullosic biomass is a complex substrate composed of crystalline cellulose, hemicellulose, and lignin. In addition to these carbon compounds, nitrogen from proteins, and other plant structures
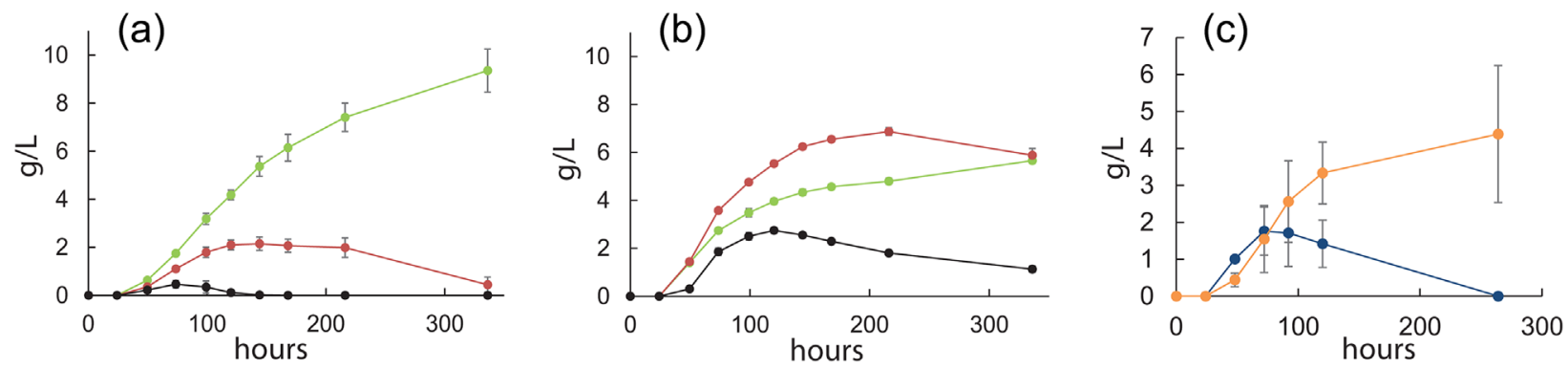

FIGURE 3 CBP conversion of MCC to organic acids by synthetic fungal consortia. (a) Glucose accumulation under low (5.88 mM, Light Green), medium (11.76 mM, Red), and high ( $23.5 \mathrm{mM}$, Black) nitrogen conditions. (b) Fumaric acid accumulation with nitrogen concentrations corresponding to (a). Error bars represent the standard deviation from four replicates. (c) Lactic acid production from $40 \mathrm{~g} / \mathrm{L} \mathrm{MCC} \mathrm{using} \mathrm{a}$ modified fungal consortium. Glucose accumulation (Blue) and lactic acid accumulation (Orange) are indicated. Error bars represent the standard deviation from two replicates 
is present in all lignocellulosic biomass. Since nitrogen concentration controls the flow of carbon between fungal growth and fumaric acid production, the amount of nitrogen added to the culture medium must complement the useable nitrogen derived from the lignocellulosic biomass substrate. The fungal consortium was seeded into RTco medium containing $20 \mathrm{~g} / \mathrm{L}$ of $\mathrm{CS}$, which is composed of $9.6 \mathrm{~g} / \mathrm{L}$ and $4.2 \mathrm{~g} / \mathrm{L}$ of glucan and xylan respectively, under three different nitrogen concentration conditions. Similar to the performance on MCC, high nitrogen conditions led to fast substrate degradation and earlier cessation of fumaric acid production compared to lower nitrogen conditions (Figure 4). The high nitrogen condition used for these experiments was $5.88 \mathrm{mM}$, much lower than in the MCC experiments, but led to similar consortium dynamics. The difference between optimal nitrogen concentrations using $\mathrm{MCC}$ versus $\mathrm{CS}$ substrates are likely due to CS-derived nitrogen. A previous study showed that similarly treated CS contained 0.6\% elemental nitrogen (Kumar, Mago, Balan, \& Wyman, 2009), which would correspond to about $9 \mathrm{mM}$ nitrogen in our cultures. It should be noted, however, only an unknown fraction of this total nitrogen can be metabolized by the fungi. A total of $0.69 \mathrm{~g} / \mathrm{L}$ of fumaric acid was produced with a yield of 0.05 by weight from total initial fermentable carbohydrates. Overall consortium performance was considerably lower compared to those for MCC as the carbon substrate. As observed in numerous previous studies, this reduction in performance is likely due to inhibitory compounds from the lignocellulosic biomass (Moreno, Ibarra, Alvira, Tomás-Pejó, \& Ballesteros, 2015). Although $R$. delemar is a promising consortium candidate because it efficiently converts sugars into fumaric acid and satisfies our major fungal consortia requirements, its acid production performance was low on CS. T. reesei was relatively much more tolerant of the CS substrate, producing $0.46 \mathrm{w} / \mathrm{w}$ yield of glucose from total initial glucan solids and $0.21 \mathrm{w} / \mathrm{w}$ yield of xylose from total initial xylan solids in monoculture (Figure 2a). Similar to approaches taken for yeast, selection of Rhizopus strains for lignocellulosic biomass tolerance may enable more efficient production (Moreno et al., 2015).

Synthetic consortia were designed to convert lignocellulosic biomass to fumaric or lactic acids. Together, $T$. reesei and $R$. delemar produced up to $6.87 \mathrm{~g} / \mathrm{L}$ fumaric acid from $40 \mathrm{~g} / \mathrm{L} \mathrm{MCC}$ in a CBP scheme without expensive supplements such as enzymes or yeast extract. Another consortium of $T$. reesei and $R$. oryzae demonstrated production of 4.4. $\mathrm{g} / \mathrm{L}$ lactic acid from MCC. Additionally, $0.69 \mathrm{~g} / \mathrm{L}$ fumaric acid was produced using CS. The rate of substrate hydrolysis was consistently higher than the rate of conversion of sugars to fumaric acid, suggesting future work to match the two rates for CBP optimization.

\section{2 | MATERIALS AND METHODS}

Trichoderma reesei strain RaVC was generously provided by (Valkonen, Penttilä, \& Benčina, 2014) of the VTT Technical Institute (Finland). Rhizopus delemar (NRRL 1526) and Rhizopus oryzae (NRRL 395) were provided by the ARS culture collection (United States Department of Agriculture). Alkaline pre-treated CS was provided by the National Renewable Energy Laboratory (Golden, CO) with the following composition of non-soluble solids: ash $7.3 \%$, ligin $17.8 \%$, glucan $47.8 \%$, xylan $21.2 \%$, galactan $1.1 \%$, arabinan $2.5 \%$, acetate $0.1 \%$ ). Slurry of the material was subjected to vacuum on Whatman \#1.1.6 ml deionized water per gram of slurry was applied to the biomass and immediately removed by vacuum filtration. The resulting biomass was dried for $48 \mathrm{hr}$ under vacuum. T. reesei, $R$. delemar, and $R$. oryzae spores were generated on potato dextrose agar (PDA) at $30^{\circ} \mathrm{C}$ for 10 days. Spores were harvested and stored in $20 \%$ glycerol at $-80^{\circ}$ $\mathrm{C}$ indefinitely. Production cultures were grown in RTco $(0.5 \mathrm{~g} / \mathrm{L}$ $\left(\mathrm{NH}_{4}\right)_{2} \mathrm{SO}_{4}, 0.125 \mathrm{~g} / \mathrm{L}$ Urea, $0.6 \mathrm{~g} / \mathrm{L} \mathrm{CaCl}_{2}, 0.4 \mathrm{~g} / \mathrm{L} \mathrm{MgSO}_{4} \times 7 \mathrm{H}_{2} \mathrm{O}$, $0.3 \mathrm{~g} / \mathrm{L} \mathrm{KH}_{2} \mathrm{PO}_{4}, 44 \mathrm{mg} / \mathrm{L} \mathrm{ZnSO}_{4} \times 7 \mathrm{H}_{2} \mathrm{O}, 10 \mathrm{mg} / \mathrm{L} \mathrm{FeSO}{ }_{4} \times 7 \mathrm{H}_{2} \mathrm{O}$, $2 \mathrm{mg} / \mathrm{L} \mathrm{CoCl}_{2} \times 6 \mathrm{H}_{2} \mathrm{O}, 1.6 \mathrm{mg} / \mathrm{L} \mathrm{MnSO}_{4} \times 4 \mathrm{H}_{2} \mathrm{O}, 0.0186 \%$ Tween-80 $[\mathrm{v} / \mathrm{v}])$ unless otherwise noted. Sterile $\mathrm{MgSO}_{4}, \mathrm{CaCl}_{2}$, and $\mathrm{FeSO}_{4}$ solutions were added immediately before culture seeding, yielding the appropriate final RTco medium concentrations, in order to prevent precipitation. Trichoderma Minimal Medium (TMM) (Minty et al., 2013) with a modified $11.76 \mathrm{mM}$ nitrogen concentration was used for lactic acid production. $T$. reesei spores from cryostock were inoculated into $10 \mathrm{ml}$ potato dextrose broth (PDB) and grown for 2 days at $30^{\circ} \mathrm{C}$ with shaking in a $50 \mathrm{ml}$ conical tube to generate a pre-culture. Mycelia from the pre-culture were pelleted at 4,600 $\mathrm{g}$ for $6 \mathrm{~min}$ and washed once in
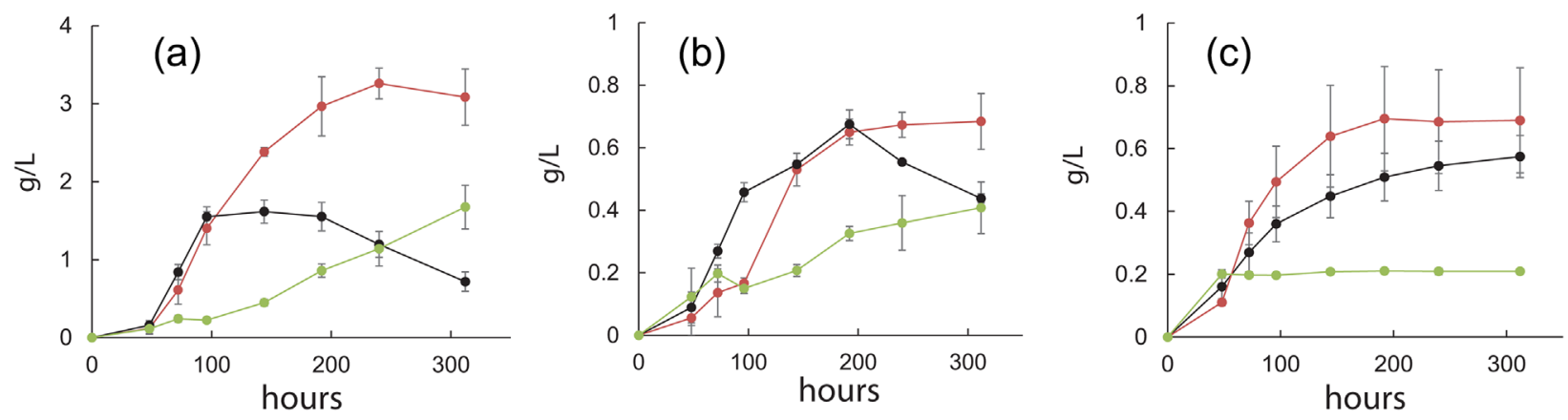

FIGURE 4 Fumaric acid production from alkaline pre-treated corn stover by fungal consortium at different nitrogen concentrations. (a) Glucose accumulation under 0 (Light Green), low (2.9 mM, Red), and high (5.88 mM, Black) added nitrogen conditions. (b) Xylose accumulation with nitrogen concentrations corresponding to (a). (c) Fumaric acid accumulation with nitrogen concentrations corresponding to (a). Nitrogen added as a medium component is lower for all corn stover conditions in comparison to MCC experiments. Error bars represent the standard deviation of four replicates 
nitrogen-free RTco medium. Two-hundren fifty microlitre of mycelia resuspended in $10 \mathrm{ml}$ of nitrogen-free RT-co medium were inoculated into $25 \mathrm{ml} \mathrm{RTco}$ medium with $20 \mathrm{~g} / \mathrm{L} \mathrm{MCC}$ and grown for 2 days in a $125 \mathrm{ml}$ baffled flask to generate an adjustment culture. The adjustment culture was used to seed production cultures at $1 \%$ of total volume. $R$. delemar or $R$. oryzae were seeded from PDA spore slants stored for less than 2 months into $100 \mathrm{ml}$ RTco medium with $20 \mathrm{~g} / \mathrm{L}$ glucose and grown for $16 \mathrm{hr}$ in a $500 \mathrm{ml}$ baffled flask with shaking to generate a preculture. Mycelia from the pre-culture were pelleted at 4,600g for $6 \mathrm{~min}$. Half of the mycelia from the resulting pellet was inoculated into $100 \mathrm{ml}$ fresh RTco medium with $3 \mathrm{~g} / \mathrm{L}$ glucose and grown for $3.5 \mathrm{hr}$ in a $500 \mathrm{ml}$ baffled flask with shaking to generate an adjustment culture. The adjustment culture was used to seed production cultures at $1 \%$ of total volume. Production cultures were grown using $25 \mathrm{~mL}$ RTco medium in $125 \mathrm{ml}$ baffled flasks at $30^{\circ} \mathrm{C}$ with $225 \mathrm{rpm}$ shaking. Sterilization of the media was achieved through autoclaving for $15 \mathrm{~min}$ at $121^{\circ} \mathrm{C}$. Glucose, fumaric acid, and lactic acid concentrations were determined by HPLC (Agilent 1100 with RID-10A detector equipped with a Rezex ${ }^{\mathrm{TM}}$ ROA-Organic Acid $\mathrm{H}+(8 \%)$ column). All reported yield and productivity values were calculated from the time point with the highest titer for the compound of interest.

\section{ACKNOWLEDGMENTS}

We thank T. Saleski, A. Krieger, and V. Sachsenhauser for valuable feedback. SAS was supported by NIH T-32-GM007315, IG by a UMEI Fellowship.

\section{ORCID}

Scott A. Scholz iD http://orcid.org/0000-0001-9168-9285

\section{REFERENCES}

Brethauer, S., \& Studer, M. H. (2014). Consolidated bioprocessing of lignocellulose by a microbial consortium. Energy and Environmental Science, 7, 1446-1453.

Carroll, A., \& Somerville, C. (2009). Cellulosic biofuels. Annual Review of Plant Biology, 60, 165-182.

den Haan, R., van Rensburg, E., Rose, S. H., Görgens, J. F., \& van Zyl, W. H. (2015). Progress and challenges in the engineering of non-cellulolytic microorganisms for consolidated bioprocessing. Current Opinion in Biotechnology, 33, 32-38.

Ding, Y., Li, S., Dou, C., Yu, Y., \& Huang, H. (2011). Production of Fumaric Acid by Rhizopus oryzae: Role of Carbon-Nitrogen Ratio. Biotechnology and Applied Biochemistry, 164, 1461-1467.

Dunn, J. B., Mueller, S., Kwon, H. Y., \& Wang, M. Q. (2013). Land-use change and greenhouse gas emissions from corn and cellulosic ethanol. Biotechnology for Biofuels, 6, 51.
Goyal, G., Tsai, S. L., Madan, B., DaSilva, N. A., \& Chen, W. (2011). Simultaneous cell growth and ethanol production from cellulose by an engineered yeast consortium displaying a functional mini-cellulosome. Microbial Cell Factories, 10, 89.

Juhász, T., Szengyel, Z., Réczey, K., Siika-Aho, M., \& Viikari, L. (2005). Characterization of cellulases and hemicellulases produced by Trichoderma reesei on various carbon sources. Process Biochemistry, 40, 3519-3525.

Kautola, H., \& Linko, Y. Y. (1989). Fumaric acid production from xylose by immobilized Rhizopus arrhizus cells. Applied Microbiology and Biotechnology, 31, 448-452.

Kawaguchi, H., Hasunuma, T., Ogino, C., \& Kondo, A. (2016). Bioprocessing of bio-based chemicals produced from lignocellulosic feedstocks. Current Opinion in Biotechnology, 42, 30-39.

Kim, S., Baek, S. H., Lee, K., \& Hahn, J. S. (2013). Cellulosic ethanol production using a yeast consortium displaying a minicellulosome and $\beta$-glucosidase. Microbial Cell Factories, 12, 14.

Kumar, R., Mago, G., Balan, V., \& Wyman, C. E. (2009). Physical and chemical characterizations of corn stover and poplar solids resulting from leading pretreatment technologies. Bioresource Technology, 100, 3948-3962.

Lambertz, C., Garvey, M., Klinger, J., Heesel, D., Klose, H., Fischer, R., \& Commandeur, U. (2014). Challenges and advances in the heterologous expression of cellulolytic enzymes: A review. Biotechnology for Biofuels, 7, 135.

Minty, J. J., Singer, M. E., Scholz, S. A., Bae, C. H., Ahn, J. H., Foster, C. E., , . Lin, X. N. (2013). Design and characterization of synthetic fungalbacterial consortia for direct production of isobutanol from cellulosic biomass. Proceedings of the National Academy of Sciences of the United States of America, 110, 14592-14597.

Moreno, A. D., Ibarra, D., Alvira, P., Tomás-Pejó, E., \& Ballesteros, M. (2015). A review of biological delignification and detoxification methods for lignocellulosic bioethanol production. Critical Reviews in Biotechnology, 35, 342-354.

Olson, D. G., McBride, J. E., Shaw, A. J., \& Lynd, L. R. (2012). Recent progress in consolidated bioprocessing. Current Opinion in Biotechnology, 23, 396-405.

Parisutham, V., Kim, T. H., \& Lee, S. K. (2014). Feasibilities of consolidated bioprocessing microbes: From pretreatment to biofuel production. Bioresource Technology, 161, 431-440.

Peterson, R., \& Nevalainen, H. (2012). Trichoderma reesei RUT-C30-thirty years of strain improvement. Microbiology, 158, 58-68.

Roa Engel, C. A., Straathof, A. J. J., Zijlmans, T. W., van Gulik, W. M., \& van der Wielen, L. A. M. (2008). Fumaric acid production by fermentation. Applied Microbiology and Biotechnology, 78, 379-389.

Valkonen, M., Penttilä, M., \& Benčina, M. (2014). Intracellular pH responses in the industrially important fungus Trichoderma reesei. Fungal Genetics and Biology, 70:86-93.

How to cite this article: Scholz SA, Graves I, Minty JJ, Lin $\mathrm{XN}$. Production of cellulosic organic acids via synthetic fungal consortia. Biotechnology and Bioengineering. 2018;115:1096-1100. https://doi.org/10.1002/bit.26509 